\title{
How to recover from a bad start: size at metamorphosis affects growth and survival in a tropical amphibian
}

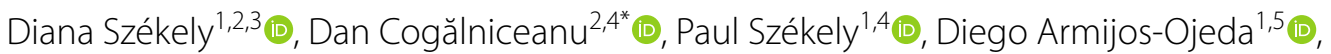 \\ Valentina Espinosa-Mogrovejo ${ }^{1}$ and Mathieu Denoël ${ }^{3}$ (1)
}

\begin{abstract}
Background: In species with complex life cycles, size at metamorphosis is a key life-history trait which reflects the complex interactions between costs and benefits of life in the aquatic and terrestrial environments. Whereas the effects of a deteriorating larval habitat (e.g. pond desiccation) on triggering an early metamorphosis have been extensively investigated in amphibians, the consequences of the resulting reduced size at metamorphosis on fitness in the post-metamorphic terrestrial stage remain poorly understood. We tested the hypothesis that a smaller size at metamorphosis negatively affects performance and survival in the ensuing terrestrial stage. Using as model a tropical amphibian (Ceratophrys stolzmanni) showing a large phenotypic plasticity in metamorphosing traits, we evaluated the effects of size at metamorphosis on fitness-related trophic and locomotor performance traits, as well as on growth and survival rates.

Results: Our results support the hypothesis that a larger size at metamorphosis is correlated with better survival and performance. The survival rate of large metamorphosing individuals was $95 \%$, compared to $60 \%$ for those completing metamorphosis at a small size. Locomotor performance and gape size were positively correlated with body size, larger animals being more mobile and capable to ingest larger prey. However, smaller individuals achieved higher growth rates, thus reducing the size gap.
\end{abstract}

Conclusions: Overall, size at metamorphosis affected profoundly the chances of survival in the short term, but smaller surviving individuals partly compensated their initial disadvantages by increasing growth rates.

Keywords: Adaptive plasticity, Growth compensation, Life cycles, Life-history, Metamorphosis

\section{Background}

Species with complex life cycles, such as biphasic amphibians and insects, are able to exploit different ecological niches and optimize their life-history in discrete developmental stages [1,2]. The transition occurring at metamorphosis usually requires dramatic and irreversible morphological transformations, and is frequently

\footnotetext{
*Correspondence: dcogalniceanu@univ-ovidius.ro

${ }^{2}$ Faculty of Natural and Agricultural Sciences, Ovidius University

Constanța, Constanța, Romania

Full list of author information is available at the end of the article
}

accompanied by a complete change of the ecological niche $[3,4]$. Pond-breeding amphibians, which in their post-larval stages become terrestrial, represent ideal models to investigate the independence of pre- and postmetamorphic life-stages and the presence of carry-over effects from one stage to the other, affecting the overall fitness of individuals [5]. The most evident trade-off between the aquatic and terrestrial stages is reflected in body size at metamorphosis. When the aquatic larvae are confronted with unfavourable conditions, such as food shortage [6, 7], high density [8], desiccation risk 
[9] or predation $[10,11]$, they can leave the aquatic environment by undergoing metamorphosis. However, this is usually done at a smaller size, which allows them to escape the immediate aquatic threats faster, but in turn exposes them to different selective pressures on land [1].

Larger size in freshly metamorphosed individuals is correlated with improved traits, like locomotor abilities and metabolic rates [12], endurance [13], resistance to desiccation [14], feeding success [15], and dispersal success [16]. Although the paradigm model [17] assumes that body size at metamorphosis is a good predictor of subsequent fitness, there are authors [e.g. 3, 18] who assert that this is not always the case. Since in amphibians a substantial percent of the adult body size is gained after metamorphosis, and because age of sexual maturity is variable, the relationship between body size at metamorphosis and fitness is more complex, especially in unpredictable environments [19]. For example, if smaller individuals have compensating mechanisms, such as more intense growth rates, metamorphic size may not have a significant effect on adult traits like mortality, age and size at first reproduction, or fecundity [20, 21]. The detrimental consequences of a small size at metamorphosis can also be compensated by changes in the morphology of juveniles [22, 23]. For example, the small froglets can have, proportionally to their body size, larger heads or longer legs than the bigger individuals. Such modifications would be beneficial because leg length influences locomotor (i.e. jumping) performance [23, 24], which in turn has been shown to positively affect food acquisition [25], predator avoidance [26] and dispersal [27]. In a similar manner, a large head width favours the swallowing ability, which is a limiting factor in prey selection $[28,29]$.

In this context, we were interested to understand the influence of size at metamorphosis on post-metamorphic traits such as survival, morphology and performance, and to investigate the existence of compensating mechanisms. Hypothesizing that the difference would be most evident between individuals at the opposite ends of the size range, we evaluated the impact of extreme size phenotypes. We selected as a model a species capable of high plasticity in metamorphosing size [30], the Pacific horned frog (Ceratophrys stolzmanni). This fossorial frog inhabits tropical dry forests, a highly seasonal ecosystem characterized by a short rainy season that lasts less than 4 months annually. The tadpoles have some of the most intense growth rates reported for anurans and can leave the water in as little as 2 weeks after egg-laying [30]. Because several environmental parameters can have interacting effects in the natural habitat [31], we used freshly metamorphosed froglets resulting from tadpoles that grew in field conditions (natural ponds) in order to cover the entire range of size at metamorphosis in the population. From the encountered spectrum of sizes, we assigned the 20 largest and 20 smallest individuals to their respective size-categories (see "Methods" section). We tested if smaller froglets can amend the detrimental survival effects induced by metamorphosing at a reduced size through (i) allometric changes in morphology or performance (wider heads, longer limbs, or better jumping skills) and/or (ii) more intense growth rates.

\section{Results}

\section{Morphology and locomotor performance}

At the completion of metamorphosis, individual body mass (BM) and snout-vent length (SVL) showed a strong correlation (Pearson's $r=0.923, n=40, P<0.001$ ), but there was no significant difference in body condition between the two categories of froglets (i.e. small vs. large: $t_{38}=0.175, P=0.862$; Additional file 1 ).

Since absolute head width was strongly correlated to SVL at metamorphosis $(r=0.982, n=40, P<0.001)$, larger froglets had significantly wider heads compared to the smaller ones $\left(t_{38}=17.123, P<0.001\right.$; Fig. 1a). Relative head width did not differ significantly between the two size categories of froglets $\left(t_{38}=-0.902, P=0.373\right)$, and was correlated with individual initial BCI $(r=0.479$, $n=40, P=0.002$ ), with froglets in better body condition having larger heads regardless of their SVL (Additional file 1).

There was a strong positive correlation between hindlimb length and SVL $(r=0.988, n=40, P<0.001)$. Compared to the large juveniles, small froglets had significantly shorter hindlimbs both in terms of absolute size $\left(t_{38}=-17.001, P<0.001\right)$, and relative size $\left(t_{38}=-6.309\right.$, $P<0.001)$. We found no relationship between the body condition of juveniles and their relative hindlimb lengths ( $r=0.114, n=40, P=0.485)$.

Absolute jumping ability was predicted by SVL $\left(F_{1,36}=102.364, \quad P<0.001\right)$, with no significant effect of either relative hindlimb length $\left(F_{1,36}=0.539\right.$, $P=0.468)$, or $\mathrm{BCI} \quad\left(F_{1,36}=0.056, P=0.815\right)$. Larger individuals were able to jump over greater distances (mean $\pm \mathrm{SE}=100.4 \pm 3.6 \mathrm{~mm}$ ) than the smaller ones $\left(59.4 \pm 2.3 \mathrm{~mm} ; t_{38}=-9.501, P<0.001\right.$; Fig. $\left.1 \mathrm{~b}\right)$.

\section{Growth}

At the end of the study period (i.e. 2 months after metamorphosis), the two categories of froglets did not differ in terms of their body condition indices (BCI; $t_{29}=-0.289$, $P=0.774$ ). Individual $\mathrm{BCI}$ after 2 months of terrestrial growth was not correlated with the froglet's SVL at metamorphosis $(r=0.107, n=31, P=0.566)$, nor with initial BCI $(r=0.191, n=31, P=0.302)$.

There was a significant effect of size at metamorphosis on post-metamorphic growth rates, with individuals that 


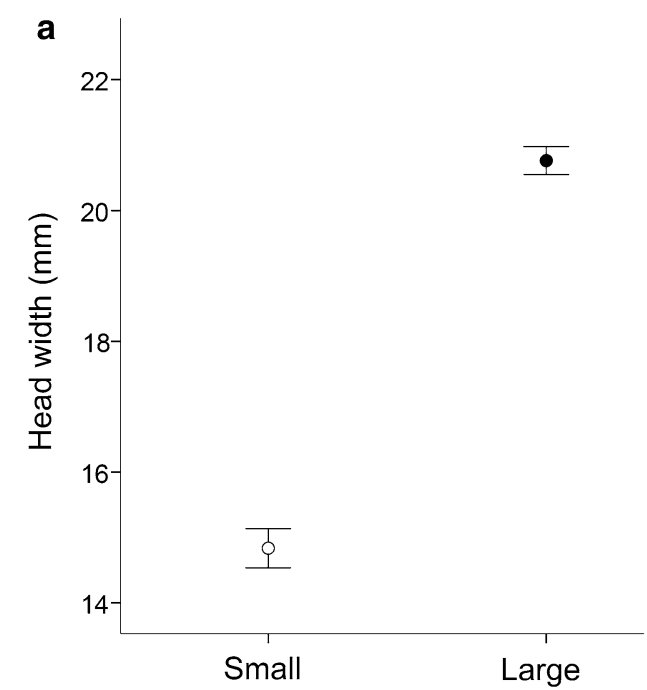

Size category assigned at metamorphosis

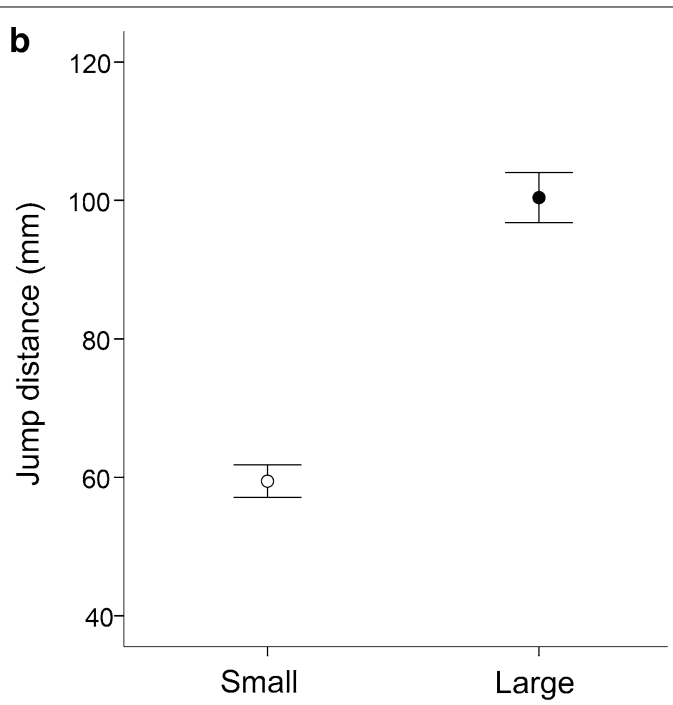

Size category assigned at metamorphosis

Fig. 1 Differences in performance between froglets of the Pacific horned frogs Ceratophrys stolzmanni which metamorphosed at small versus large body size (developmental stage Gosner 46; mean $\pm \mathrm{SE}$ ): $\mathbf{a}$ head width (gape size) and $\mathbf{b}$ jump distance

metamorphosed at a smaller size having a higher increase in SVL, both in absolute values $\left(r^{2}=0.31, F_{1,29}=13.054\right.$, $P=0.001$ ), and in percentage gained by the end of the study $\left(r^{2}=0.566, F_{1,29}=37.782, P<0.001\right.$, Fig. 2 a). This means that individuals metamorphosing at a small size grew at a significantly higher rate than those metamorphosing at a large size $(0.08 \mathrm{~mm} /$ day versus $0.06 \mathrm{~mm} /$ day on average, respectively; $t_{29}=2.29, P=0.03$ ). In spite of this, the differences in SVL between the two categories of froglets remained significant until the end of the study $\left(t_{29}=-13.089, P<0.001\right)$, although the magnitude decreased (the difference between average SVL in the two groups decreasing from $39 \%$ at the start of the study to $27 \%$ at the end).

\section{Mortality}

Survivorship over the 2-month study was significantly different between the two categories of froglets: $95 \%$ (19 out of 20) of the large individuals survived until the end of the study (i.e. 62 days), compared to $60 \%$ (12 out

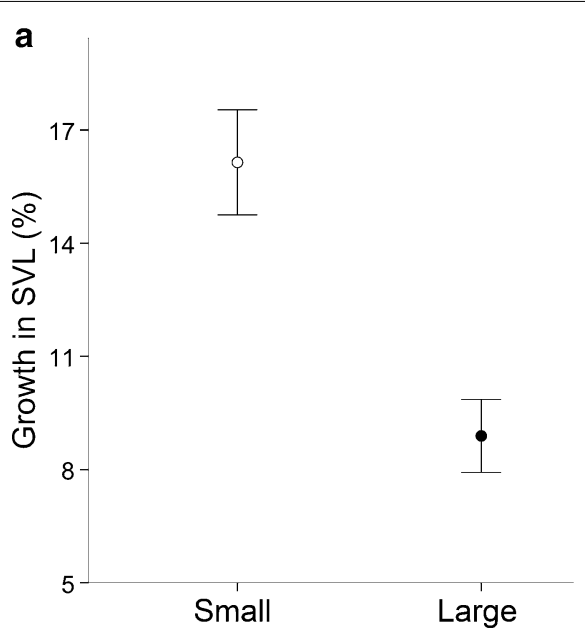

Size category asigned at metamorphosis

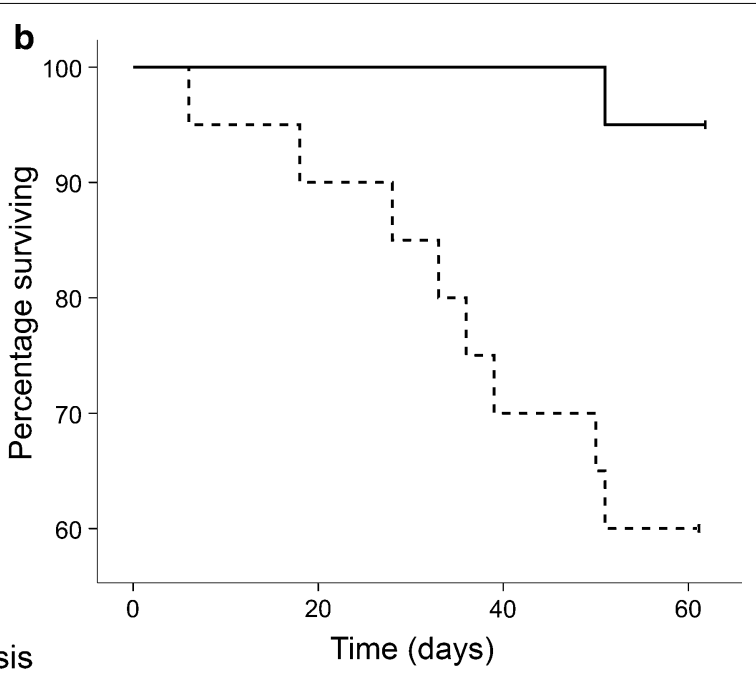

Fig. 2 a Growth (mean \pm SE increase in SVL) of juveniles of Pacific horned frogs Ceratophrys stolzmanni that metamorphosed at a small versus a large body size over the study period following metamorphosis (62 days); b Kaplan-Meier survival curves for the two groups of juveniles after metamorphosis. Dotted line: small metamorphosing juveniles; full line: large metamorphosing juveniles 
of 20) of the small individuals (log-rank test, $X^{2}=7.318$, $P=0.007$; Fig. $2 b$ ). The BCI at metamorphosis was not a predictor of mortality (Cox regression, $X^{2}=0.154$, d.f. $=1, P=0.695)$.

\section{Discussion}

Life-history switch-points such as metamorphosis, that require major and irreversible changes in morphology, anatomy, physiology, and habitat and resource use, have a profound effect on individual fitness and involve tradeoffs $[17,32,33]$. Our study reveals that individuals making the transition from aquatic larvae to the terrestrial stage at a large size experience higher survival rates during the first activity season. Even under favourable experimental conditions, with no predation or competition, and ad libitum food resources, the juveniles that metamorphosed at a small size had a higher mortality rate. It is expected that in the natural environment, smaller size would result in a further increase in mortality due to exposure to predators [34] and desiccation [14]. Our results imply that, although developmental plasticity can allow tadpoles to escape an unfavourable aquatic environment (e.g. drying pond, high density, reduced food availability) before reaching an optimal size, thus avoiding mortality in the larval stage, it has a direct cost on survival in the terrestrial stage. The lower survival rate of smaller juveniles in terrestrial habitat is consistent with observations made by some authors $[6,15]$, although in other cases no significant long-term benefits for larger size at metamorphosis in terms of survival was found [21, 35].

Although size at metamorphosis is predicted to have a large impact on size at maturity [32], in some species it was shown that, if environmental conditions are optimal for growth, smaller juveniles can compensate by growing faster and the differences in size eventually fade away $[7,36]$. Indeed, our study showed that individuals metamorphosing at a small size are able to increase their growth rate compared to larger individuals, and thus diminish the size gap over time. However, long-term capture-mark-recapture surveys are needed to confirm this pattern in natural conditions.

The existence of compensatory growth (i.e. an increase in growth rates once the conditions are favourable in order to compensate partly or completely the deprivations experienced in younger stages) has been reported in various taxa [37] and can be the result of behavioural modifications, such as prolonged foraging activity and higher ingestion rates $[6,38,39]$, or morphological adaptations that allow digestion of larger quantities of food [40]. However, a higher than optimal growth rate comes at a fitness cost, reflected in decreased survival [37]. Along with ecological factors, such as increased exposure to predators and competitors because of prolonged foraging [38], several other components can contribute to the lower fitness associated with accelerated growth rates, amongst which a lower resistance to starvation because of intense metabolism and low lipid reserves [40], delayed ossification [41], depressed immunological function [42], or cellular oxidative stress [43]. Some of the intrinsic costs mentioned above might have contributed to the observed higher mortality in the froglets that had metamorphosed at a small size in our study.

Pacific horned frogs showed one of the widest range of sizes at metamorphosis reported for any amphibian in their natural population, individuals differing by up to $100 \%$ in body size and $890 \%$ in body mass. This range is broader than previously reported intrapopulation variation in the literature [31, 43]. Pond permanence [30], food availability [44], temperature [45] and presence of predators or competitors $[46,47]$ are known to have a profound effect on individual metamorphosing size in anurans, and the interaction between various selective pressures can determine a large spectrum of sizes. Additionally, in the case of anuran species reproducing in ephemeral ponds, individuals are less capable to delay their metamorphosis or further increase their larval growth rates, which are already close to the physiological limit [48]. This implies that differences in larval environment will produce a large variation in metamorphic body sizes, such as the one we report here. In the natural habitat, a high diversity of sizes is likely to reduce food competition amongst froglets and allow for a more rapid growth for both large and small individuals [49].

Differences in shape at metamorphosis (i.e. size of different structures in relation to total individual size) are attributed to environmental conditions that promote variations in larval growth rates $[23,50]$. Studies carried out on various species give contrasting results, especially in the case of the relationship between hind-limb size (or segments of it) and the individual size [22]. For example, temperature-induced intensification of larval growth rates can generate individuals with relatively shorter legs $[22,24]$ or longer legs [51]; desiccation-triggered acceleration of growth can determine relatively shorter legs [23], while presence of predators and lack of food can determine slower growth correlated with relatively shorter legs [11]. In our case, metamorphosing at a smaller size was associated with shorter hindlimbs, not only in absolute, but even in relative terms, indicating a departure from isometric size of limbs in this species. Such shorter legs have also been shown to be a result of accelerated development in other species $[11,52]$.

However, we found that the differences in relative size of hindlimbs did not affect jumping ability in the case of horned frogs; the main factor determining locomotor 
performance was the body size of juveniles. In fossorial frogs, the leg length in relation to the body size is probably less flexible compared to non-fossorial species of frogs $[24,53]$, as a result of managing the contrasting selective pressures of avoiding predation or desiccation and efficient burrowing [54]. Alternatively, the variation of leg length might have been too small to determine a measurable difference in jumping distance [55]. We found locomotor performance to be strictly size-dependent, larger individuals having longer legs and jumping over larger distances in response to a simulated predator attack. In anurans, jumping ability is directly related to individual fitness, allowing predator avoidance [26, 34], prey acquisition [25] and dispersal [27].

For gape-limited predators such as amphibians, the width of the head is another morphological trait that can improve fitness, by permitting individuals with wider gape access to larger food items [56]. Since tadpoles of Ceratophryidae are macrophagous, and because their mouth parts go through relatively little restructuration at metamorphosis [57], it can be assumed that the larger relative head width can be an explanation for the improved individual body condition at metamorphosis, regardless of the body size. Overall, head width after metamorphosis was proportional to individual SVL. In the case of strong intra- and inter-specific competition or food shortage, the larger individuals may thus be better equipped for prey ingestion compared to smaller individuals by having access to a wider range of prey sizes. Additionally, since Ceratophrys species are known to prey on other amphibians [58], small individuals would also be more at risk of being victims of cannibalistic events. In the cane toad (Rhinella marina), the victims of cannibalistic events were a non-random subset of the juvenile population, represented by the smallest and weakest individuals [59]. The lack of beneficial modifications in the proportions of investigated anatomical features, together with the effect of size are likely to act synergistically in nature, decreasing the chances of survival of small metamorphosing individuals, especially in areas with a high density of individuals that increase the probability of cannibalistic encounters.

\section{Conclusions}

Our study links two stages (i.e. aquatic and terrestrial) in amphibian life-history, helping to understand how size at metamorphosis, which is determined by conditions in the aquatic environment, affects the subsequent terrestrial stage, impacting the success and survival of individuals and potentially influencing population dynamics. When aquatic conditions deteriorate, faster metamorphosis is the best survival option for tadpoles, allowing them to take advantage of the terrestrial habitat, where they are able to compensate for smaller size at metamorphosis with an increased growth rate. However, there is a trade-off, as the benefits of leaving water early in life are offset by a lower survival.

\section{Methods}

Study site and sampling. On the 16-17 April 2015, we collected 92 freshly metamorphosed C. stolzmanni, all in developmental stage Gosner 45 [mouth angle at level of posterior margin of the eye, tail reduced to a stub, 60] in Arenillas Ecological Reserve (03 $34^{\prime} \mathrm{S} ; 80^{\circ} 08^{\prime} \mathrm{E}, 30 \mathrm{~m}$ a.s.l.), southern Ecuador. This stage was chosen because it allows to select individuals that just exited water at metamorphosis. Because mating is generally synchronized to one night in the studied population [61], we considered that all froglets had approximately the same age. The froglets $(n=92)$ were found in terrestrial habitats, in an area within a 50-m radius from ponds used for reproduction. The average snout-vent length $(\mathrm{SVL} \pm \mathrm{SD})$ of froglets was $34.5 \pm 4.9 \mathrm{~mm}$ (range: $23.8-47.9 \mathrm{~mm}$ ), and average body mass $(B M \pm S D)$ was $4.1 \pm 1.9 \mathrm{~g}$ (range: $1.2-11.9 \mathrm{~g}$ ). From this spectrum of sizes (Additional file 2), we selected the extreme phenotypes, i.e. the smallest 20 individuals $(\mathrm{SVL}=28.21 \pm 1.9 \mathrm{~mm}$, range $23.8-30.3 \mathrm{~mm} ; \mathrm{BM}=2.1 \pm 0.6 \mathrm{~g}$, range $1.2-3.6)$ and the largest 20 individuals $(\mathrm{SVL}=41.1 \pm 2.7 \mathrm{~mm}$, range $38.3-$ $47.9 \mathrm{~mm} ; \mathrm{BM}=6.7 \pm 1.6 \mathrm{~g}$, range $5.1-11.9 \mathrm{~g}$ ).

\section{Animal care}

We raised the froglets in an outdoor laboratory for a period of 62 days (until 24 June 2015), mimicking the duration of an activity season in the natural environment, until the aestivation period (during the dry season), which begins approximately at the end of June (pers. obs.). The froglets were kept individually, in mesh-covered (to prevent escape) plastic tanks $(21 \times 15 \mathrm{~cm}, 12 \mathrm{~cm}$ high), with a 5-cm layer of moist soil that allowed natural burrowing behaviour [54]. Temperature, relative humidity and light regime were similar to the ones in natural habitat, except for a roof that provided protection from direct sun heating. All tanks were kept in similar conditions, and their relative position was randomly changed after each feeding. The animals were fed an ad libitum, appropriately sized, diet of crickets (similar mixed sizes items for both frog size groups at any time, overall size of crickets slightly larger as the froglets grew). Uneaten insects were removed and fresh food was added every day for the first month, and afterwards every other day until the end of the study. The tanks were checked daily for potential occurrence of death of individuals and sprayed with water to maintain soil humidity. After the completion of the study, on 25 June 2015, all froglets were released at the capture site. 


\section{Morphological traits}

The morphometric parameters used in the analyses were SVL (snout-vent length, from the tip of the snout to the cloaca), BM (body mass), head width (at the corners of the mouth), hindlimb length [total hindlimb length, measured as the sum of the right femur, tibia, metatarsus, and the length between the end of the metatarsus to the end of digit IV, 62]. Total hindlimb length in amphibians and its size relative to the total size of the animal is a morphometric trait influencing locomotor ability [63]. Measurements were taken at the beginning (on the 23 April 2015, in G46 developmental stage, i.e. after the complete resorption of the tail so that it does not interfere with movement) and at the end of the study (62 days after the start of the experiment - SVL and BM only), using a Dial-Max calliper $(0.1 \mathrm{~mm}$ precision) and a My Weigh $300 Z$ portable scale ( 0.1 g precision). Daily growth rates were calculated as (final SVL - initial SVL)/62. Individuals that died during the study $(n=9)$ were excluded from the growth rate analysis.

\section{Locomotor performance}

Jumping performance tests were taken on 23 April 2015, when individuals had reached developmental stage G46. During the 2 days prior to these trials, the juveniles did not receive any food, so that the presence of food in the digestive system would not influence the results [53]. The lack of feeding during metamorphosis is normal in anurans, due to changes in their digestive system [64]. Jumping performance trials were carried out at a mean $\pm S E$ of $22 \pm 1{ }^{\circ} \mathrm{C}$, at night-time (21:00-24:00, local time, i.e. during the normal activity hours for the species). Each tested individual $(n=40)$ was placed in the centre of a plastic arena $(75 \times 45 \mathrm{~cm}, 33 \mathrm{~cm}$ high), lined with a $5 \mathrm{~cm}$ layer of moist soil, to provide natural adherence and favour movement [36]; the soil layer being replaced between each trial. Movement (i.e. jumping) was elicited by a gentle touching of the urostyle with a long probe $(50 \mathrm{~cm}$ long, $1 \mathrm{~mm}$ diameter metal stick, with rounded tip), at three times, with a 1-min break between two successive trials. The juvenile was replaced in the centre of the arena before each trial. In the rare occasions when touching elicited a series of multiple movements, only the first jump was measured. The final jumping distance for each individual was the maximum value of the three trials.

\section{Statistical analysis}

Data analysis was carried out with SPSS 21.0 (IBM Corp., Armonk, NY), with a significance level set at 0.05 (dataset available in Additional file 3). Values are given as mean $\pm \mathrm{SE}$. All data were first assessed for normality (QQ-plot inspection) and homogeneity of variance (Levene test, $P>0.05$ ); some variables (SVL, $\mathrm{BM}$, head width and hindlimb length) were squaretransformed to fit the normality assumption. The correlations between various morphological variables were assessed with Pearson's $r$ correlation tests. Linear regression ( $F$ test) was used to test the effect of size at metamorphosis on growth rates (absolute, percentual) of the froglets. Body condition indexes (BCI) were computed as residuals of $\operatorname{lnBM}$ against $\ln S V L$ [65]; BCI is considered a good estimate of lipid storage in amphibians [66]. We calculated a relative hindlimb length as the ratio between hindlimb length and SVL, as this was shown to be related to jumping ability [67], and the relative head width as the ratio between head width and SVL. Comparisons of various relevant traits (BCI, head width, hindlimb length, growth rates) between the two size-categories of froglets were investigated using Student's $t$-tests. To assess if size-free changes in leg size can influence jumping ability, general linear models were built using SVL and size independent traits (relative hindlimb length and $\mathrm{BCI}$ ) as explanatory variables, and jumping ability as the dependent variable.

Survival rates were calculated as the percentage of individuals that survived until the end of the study in each size-group. The log-rank (Mantel-Cox) test based on Kaplan-Meier survival curves was used to detect differences in survivorship among the two groups, while the influence of body condition on survival probability was tested through a Cox proportional-hazards regression model.

\section{Supplementary information}

Supplementary information accompanies this paper at https://doi. org/10.1186/s12898-020-00291-w.

Additional file 1. Morphometry, locomotor performance traits and growth rates for individuals from the two "metamorphosis" size groups (SVL: snout-vent length). Initial measurement of morphometric traits, as well as jumping trials, were performed in Gosner 46 developmental stage, while final ones were taken 62 days after metamorphosis.

Additional file 2. Difference in size at metamorphosis in Pacific horned frogs Ceratophrys stolzmanni (developmental stage Gosner 45), $n=92$. The 20 smallest (white circles) and 20 largest individuals (black dots) were selected to be used in the experiment. The small insert shows a large and a small froglet (photos Diana Székely).

Additional file 3. Raw data.

\section{Abbreviations}

SVL: Snout-vent length; BM: Body mass; BCl: Body condition index.

\section{Acknowledgements}

Fieldwork in the reserve was possible thanks to the help of the Arenillas Ecological Reserve administration. We are especially grateful to the REA "guardaparques" for their friendship and support during the fieldwork. 


\section{Authors' contributions}

DS and PS designed the study. DS, PS, DAO and VEM conducted fieldwork and performed the experiments. DS analyzed the data. DS, DC, PS, DAO and MD wrote the manuscript. All authors read and approved the final manuscript.

\section{Funding}

DSz benefited from a research Grant from Universidad Técnica Particular de Loja (Convocatoria Estancias Cortas de Investigacion 2019). The collaboration between Ovidius University Constanta and University of Liège was supported by Wallonie-Bruxelles International (W.B.I.) and CCCDI-UEFISCDI (A.N.C.S.: Autoritatea Nationala pentru Cercetare Stiintifica)-project no. 105 BM/2017. $\mathrm{MD}$ is a Research Director at Fonds de la Recherche Scientifique-FNRS (Belgium).

\section{Availability of data and materials}

All data generated or analysed during this study are included in this published article and its supplementary information files (Additional files 1,2 and 3).

\section{Ethics approval and consent to participate}

Research permit was issued by Ministerio del Ambiente del Ecuador (MAEDNB-CM-2015-0016, granted to Universidad Técnica Particular de Loja). All applicable institutional and/or national guidelines for the care and use of animals were followed. The study was approved by the UTPL Ethics Committee (UTPL-CBEA-2016-001).

\section{Consent for publication}

Not applicable.

\section{Competing interests}

The authors declare that they have no competing interests.

\section{Author details}

${ }^{1}$ Departamento de Ciencias Biológicas, EcoSs Lab, Universidad Técnica Particular de Loja, Loja, Ecuador. ${ }^{2}$ Faculty of Natural and Agricultural Sciences, Ovidius University Constanța, Constanța, Romania. ${ }^{3}$ Laboratory of Ecology and Conservation of Amphibians (LECA), Freshwater and OCeanic Science Unit of ReSearch (FOCUS), University of Liège, Liège, Belgium. ${ }^{4}$ Asociation Chelonia, Bucharest, Romania. ${ }^{5}$ Programa de Doctorado en Conservación de Recursos Naturales, Universidad Rey Juan Carlos, Móstoles, Spain.

Received: 24 January 2020 Accepted: 11 April 2020

Published online: 21 April 2020

\section{References}

1. Wilbur HM. Complex life cycles. Annu Rev Ecol Syst. 1980;11:67-93. https ://doi.org/10.1146/annurev.es.11.110180.000435.

2. Laudet $\mathrm{V}$. The origins and evolution of vertebrate metamorphosis. Curr Biol. 2011;21:726-37. https://doi.org/10.1016/j.cub.2011.07.030.

3. Rowe L, Ludwig D. Size and timing of metamorphosis in complex life cycles: time constraints and variation. Ecology. 1991;72:413-27. https:// doi.org/10.2307/2937184.

4. Hardy NB, Peterson DA, Dohlen CD. The evolution of life cycle complexity in aphids: ecological optimization or historical constraint? Evolution. 2015;69:1423-32. https://doi.org/10.1111/evo.12643.

5. Tomašević Kolarov N, Cvijanović M, Denoël M, Ivanović A. Morphological integration and alternative life history strategies: a case study in a facultatively paedomorphic newt. J Exp Zool B Mol Dev Evol. 2017;328:737-48. https://doi.org/10.1002/jez.b.22758.

6. Morey S, Reznick D. Effects of larval density on postmetamorphic spadefoot toads (Spea hammondii). Ecology. 2001;82:510-22. https://doi. org/10.1890/0012-9658(2001)082\%5b0510:EOLDOP\%5d2.0.CO;2.

7. Crespi EJ, Warne RW. Environmental conditions experienced during the tadpole stage alter post-metamorphic glucocorticoid response to stress in an amphibian. Integr Comp Biol. 2013;53:989-1001. https://doi. org/10.1093/icb/ict087.

8. Yagi KT, Green DM. Mechanisms of density-dependent growth and survival in tadpoles of Fowler's toad, Anaxyrus fowleri: volume vs. abundance. Copeia. 2016;104:942-51. https://doi.org/10.1643/CE-16-438.
9. Székely $P$, Tudor M, Cogălniceanu D. Effect of habitat drying on the development of the Eastern spadefoot toad (Pelobates syriacus) tadpoles. Amphibia-Reptilia. 2010;31:425-34. https://doi.org/10.1163/1568538107 91769536.

10. Skelly DK, Werner EE. Behavioral and life-historical responses of larval American toads to an odonate predator. Ecology. 1990;71:2313-22. https ://doi.org/10.2307/1938642.

11. Van Buskirk J, Saxer G. Delayed costs of an induced defense in tadpoles? Morphology, hopping, and development rate at metamorphosis. Evolution. 2001;55:821-9. https://doi.org/10.1111/j.0014-3820.2001.tb00817.x.

12. Beck CW, Congdon JD. Effects of age and size at metamorphosis on performance and metabolic rates of southern toad, Bufo terrestris, metamorphs. Funct Ecol. 2000;14:32-8. https://doi.org/10.104 6/j.1365-2435.2000.00386.x.

13. John-Alder HB, Morin PJ. Effects of larval density on jumping ability and stamina in newly metamorphosed Bufo woodhousii fowleri. Copeia. 1990;1990:856-60. https://www.jstor.org/stable/1446453.

14. Child T, Phillips BL, Brown GP, Shine R. The spatial ecology of cane toads (Bufo marinus) in tropical Australia: why do metamorph toads stay near the water? Austral Ecol. 2008;33:630-40. https://doi.org/10.111 1/j.1442-9993.2007.01829.x.

15. Cabrera-Guzmán E, Crossland MR, Brown GP, Shine R. Larger body size at metamorphosis enhances survival, growth and performance of young cane toads (Rhinella marina). PLoS ONE. 2013;8:e70121. https://doi. org/10.1371/journal.pone.0070121.

16. Chelgren ND, Rosenberg DK, Heppell SS, Gitelman Al. Carryover aquatic effects on survival of metamorphic frogs during pond emigration. Ecol Appl. 2006;16:250-61. https://doi.org/10.1890/04-0329.

17. Wilbur HM, Collins JP. Ecological aspects of amphibian metamorphosis. Science. 1973;182:1305-14. https://doi.org/10.1126/scien ce.182.4119.1305.

18. Werner EE. Amphibian metamorphosis: growth rate, predation risk, and the optimal size at transformation. Am Nat. 1986;128:319-41. https://doi. org/10.1086/284565.

19. Earl JE, Whiteman $\mathrm{HH}$. Are commonly used fitness predictors accurate? A meta-analysis of amphibian size and age at metamorphosis. Copeia. 2015;103:297-309. https://doi.org/10.1643/CH-14-128.

20. Boone MD. Juvenile frogs compensate for small metamorph size with terrestrial growth: overcoming the effects of larval density and insecticide exposure. J Herpetol. 2005;39:416-23. https://doi.org/10.1670/187-04A.1.

21. Schmidt BR, Hödl W, Schaub M. From metamorphosis to maturity in complex life cycles: equal performance of different juvenile life history pathways. Ecology. 2012;93:657-67. https://doi.org/10.1890/11-0892.1.

22. Gómez-Mestre I, Saccoccio VL, lijima T, Collins EM, Rosenthal GG, Warkentin KM. The shape of things to come: linking developmental plasticity to post-metamorphic morphology in anurans. J Evol Biol. 2010;23:1364-73. https://doi.org/10.1111/j.1420-9101.2010.02016.x.

23. Charbonnier JF, Vonesh JR. Consequences of life history switch point plasticity for juvenile morphology and locomotion in the Túngara frog. PeerJ. 2015;3:e1268. https://doi.org/10.7717/peerj.1268.

24. Ficetola FG, de Bernardi F. Trade-off between larval development rate and post-metamorphic traits in the frog Rana latastei. Evol Ecol. 2006;20:14358. https://doi.org/10.1007/s10682-005-5508-6.

25. Walton M. Relationships among metabolic, locomotory, and field measures of organismal performance in the Fowler's toad (Bufo woodhousei fowleri). Physiol Zool. 1988;61:107-18. https://doi.org/10.1086/physz ool.61.2.30156141.

26. Wassersug RJ, Sperry DG. The relationships of locomotion to differential predation on Pseudacris triseriata (Anura: Hylidae). Ecology. 1977;58:8309. https://doi.org/10.2307/1936218.

27. Phillips BL, Brown GP, Webb JK, Shine R. Invasion and the evolution of speed in toads. Nature. 2006;439:803. https://doi.org/10.1038/439803a.

28. Emerson SB. Skull shape in frogs: correlations with diet. Herpetologica. 1985:41:177-88.

29. Denoël M. Feeding performance in heterochronic alpine newts is consistent with trophic niche and maintenance of polymorphism. Ethology. 2004;110:127-36. https://doi.org/10.1111/j.1439-0310.2003.00958.x.

30. Székely D, Denoël M, Székely P, Cogălniceanu D. Pond drying cues and their effects on growth and metamorphosis in a fast developing amphibian. J Zool. 2017;303:129-35. https://doi.org/10.1111/jzo.12468. 
31. Grözinger F, Thein J, Feldhaar H, Rödel M-O. Giants, dwarfs and the environment-metamorphic trait plasticity in the common frog. PLOS ONE. 2014;9:e89982. https://doi.org/10.1371/journal.pone.0089982.

32. Altwegg R, Reyer HU. Patterns of natural selection on size at metamorphosis in water frogs. Evolution. 2003;57:872-82. https://doi. org/10.1111/j.0014-3820.2003.tb00298.x.

33. Rudolf VHW, Rödel MO. Phenotypic plasticity and optimal timing of metamorphosis under uncertain time constraints. Evol Ecol. 2007;21:121-42. https://doi.org/10.1007/s10682-006-0017-9.

34. Ward-Fear G, Brown GP, Shine R. Factors affecting the vulnerability of cane toads (Bufo marinus) to predation by ants. Biol J Linn Soc. 2010;99:738-51. https://doi.org/10.1111/j.1095-8312.2010.01395.x.

35. Smith DC. Adult recruitment in chorus frogs: effects of size and date at metamorphosis. Ecology. 1987;68:344-50. https://doi.org/10.2307/19392 65.

36. Bredeweg EM, Urbina J, Morzillo AT, Garcia TS. Starting on the right foot: carryover effects of larval hydroperiod and terrain moisture on postmetamorphic frog movement behavior. Front Ecol Evol. 2019;7:97. https ://doi.org/10.3389/fevo.2019.00097.

37. Hector KL, Nakagawa S. Quantitative analysis of compensatory and catchup growth in diverse taxa. J Anim Ecol. 2012;81:583-93. https://doi.org/10 $.1111 / j .1365-2656.2011 .01942 . x$

38. Tarvin RD, Silva Bermúdez C, Briggs VS, Warkentin KM. Carry-over effects of size at metamorphosis in red-eyed treefrogs: higher survival but slower growth of larger metamorphs. Biotropica. 2015;47:218-26. https://doi. org/10.1111/btp.12198.

39. Buhaciuc E, Székely P, Băncilă R, Cogălniceanu D. Food availability influences postmetamorphic growth in two spadefoot toad species (genus Pelobates). Amphib Reptil. 2017;38:41-8. https://doi.org/10.1163/15685 381-00003082.

40. Bouchard SS, O'Leary CJ, Wargelin L, Charbonnier JF, Warkentin KM. Postmetamorphic carry-over effects of larval digestive plasticity. Funct Ecol. 2016;30:379-88. https://doi.org/10.1111/1365-2435.12501.

41. Arendt JD, Wilson DS. Population differences in the onset of cranial ossification in pumpkinseed (Lepomis gibbosus), a potential cost of rapid growth. Can J Fish Aquat Sci. 2000;57:351-6. https://doi.org/10.1139/ f99-250.

42. Gervasi SS, Foufopoulos J. Costs of plasticity: responses to desiccation decrease post-metamorphic immune function in a pondbreeding amphibian. Funct Ecol. 2008;22:100-8. https://doi.org/10.111 1/j.1365-2435.2007.01340.x.

43. Burraco P, Díaz-Paniagua C, Gomez-Mestre I. Different effects of accelerated development and enhanced growth on oxidative stress and telomere shortening in amphibian larvae. Sci Rep. 2017;7:7494. https:// doi.org/10.1038/s41598-017-07201-z.

44. Audo MC, Mann TM, Polk TL, Loudenslager CM, Diehl WJ, Altig R. Food deprivation during different periods of tadpole (Hyla chrysoscelis) ontogeny affects metamorphic performance differently. Oecologia. 1995;103:518-22. https://doi.org/10.1007/BF00328691.

45. Walsh PT, Downie JR, Monaghan P. Temperature-mediated morphology changes during metamorphic climax in the African clawed frog, Xenopus laevis. J Therm Biol. 2008;33:244-9. https://doi.org/10.1016/j.jther bio.2008.02.002.

46. Laurila A. Competitive ability and the coexistence of anuran larvae in freshwater rock-pools. Freshwater Biol. 2000;43:161-74.

47. Davenport JM, Hossack BR, Lowe WH. Partitioning the non-consumptive effects of predators on prey with complex life histories. Oecologia. 2014;176:149-55. https://doi.org/10.1007/s00442-014-2996-5.

48. Richter-Boix A, Tejedo M, Rezende EL. Evolution and plasticity of anuran larval development in response to desiccation. A comparative analysis. Ecol Evol. 2011;1:15-25. https://doi.org/10.1002/ece3.2.

49. Benard MF, Middlemis Maher J. Consequences of intraspecific niche variation: phenotypic similarity increases competition among recently metamorphosed frogs. Oecologia. 2011;166:585-92. https://doi.org/10.1007/ s00442-010-1896-6.
50. Tejedo M, Marangoni F, Pertoldi C, Richter-Boix A, Laurila A, Orizaola G, Nicieza AG, Álvarez D, Gomez-Mestre I. Contrasting effects of environmental factors during larval stage on morphological plasticity in postmetamorphic frogs. Clim Res. 2010;43(1):31-9. https://doi.org/10.3354/ cr00878.

51. Blouin SM, Brown TS. Effects of temperature-induced variation in anuran larval growth rate on head width and leg length at metamorphosis. Oecologia. 2000;125:358-61. https://doi.org/10.1007/s004420000458.

52. Gómez-Mestre I, Buchholz DR. Developmental plasticity mirrors differences among taxa in spadefoot toads linking plasticity and diversity. PNAS. 2006;103:19021-6. https://doi.org/10.1073/pnas.0603562103.

53. Tejedo M, Semlitsch RD, Hotz H. Differential morphology and jumping performance of newly metamorphosed frogs of the hybridogenetic Rana esculenta complex. J Herpetol. 2000;34:201-10. https://doi. org/10.2307/1565416.

54. Székely D, Cogălniceanu D, Székely P, Denoël M. Dryness affects burrowing depth in a semi-fossorial amphibian. J Arid Environ. 2018;155:79-81. https://doi.org/10.1016/j.jaridenv.2018.02.003.

55. Emerson SB. Allometry and jumping in frogs: helping the twain to meet. Evolution. 1978;32:551-64. https://doi.org/10.1111/j.1558-5646.1978. tb04598.x.

56. Hirai T. Ontogenetic change in the diet of the pond frog, Rana nigromaculata. Ecol Res. 2002;17:639-44. https://doi.org/10.104 6/j.1440-1703.2002.00521.x.

57. Fabrezi M, Quinzio SI, Goldberg J, Cruz JC, Pereyra MC, Wassersug RJ. Developmental changes and novelties in ceratophryid frogs. EvoDevo. 2016;7:1-16. https://doi.org/10.1186/s13227-016-0043-9.

58. Székely D, Gaona FP, Székely P, Cogălniceanu D. What does a Pacman eat? Macrophagy and necrophagy in a generalist predator (Ceratophrys stolzmanni). PeerJ. 2019;7:e6406. https://doi.org/10.7717/peerj.6406.

59. Pizzatto L, Shine R. The behavioral ecology of cannibalism in cane toads (Bufo marinus). Behav Ecol Sociobiol. 2008;63:123-33. https://doi. org/10.1007/s00265-008-0642-0.

60. Gosner KL. A simplified table for staging anuran embryos and larvae with notes on identification. Herpetologica. 1960;16:183-90.

61. Székely D, Székely P, Denoël M, Cogălniceanu D. Random size-assortative mating despite size-dependent fecundity in a Neotropical amphibian with explosive reproduction. Ethology. 2018;124:218-26. https://doi. org/10.1111/eth.12724.

62. Buckley CR, Michael SF, Irschick DJ. Early hatching decreases jumping performance in a direct-developing frog, Eleutherodactylus coqui. Funct Ecol. 2005; 19:67-72.

63. Rebelo AD, Measey J. Locomotor performance constrained by morphology and habitat in a diverse clade of African frogs (Anura: Pyxicephalidae). Biol J Linn Soc. 2019;127:310-23. https://doi.org/10.1093/biolinnean /blz007.

64. Hourdry J, I'Hermite A, Ferrand R. Changes in the digestive tract and feeding behavior of anuran amphibians during metamorphosis. Physiol Zool. 1996;69:219-51.

65. Băncilă RI, Hartel T, Plăiaşu R, Smets J, Cogălniceanu D. Comparing three body condition indices in amphibians: a case study of yellow-bellied toad Bombina variegata. Amphib Reptil. 2010;31:558-62. https://doi. org/10.1163/017353710X518405.

66. Denoël M, Hervant F, Schabetsberger R, Joly P. Short- and long-term advantages of an alternative ontogenetic pathway. Biol J Linn Soc. 2002;77:105-12. https://doi.org/10.1046/j.1095-8312.2002.00095.x.

67. Zug GR. Anuran locomotion: structure and function. I. Preliminary observations on relation between jumping and osteometrics of appendicular and postaxial skeleton. Copeia. 1972;1972:613-24. https://doi. org/10.2307/1442720.

\section{Publisher's Note}

Springer Nature remains neutral with regard to jurisdictional claims in published maps and institutional affiliations. 